\title{
ON SPACES IN WHICH TWO POINTS DETERMINE A GEODESIC
}

\author{
BY \\ HERBERT BUSEMANN
}

The present note deals with spaces in which geodesics exist and are uniquely determined by any two of their points.

The existence of geodesics without additional topological or differentiability conditions finds its expression in the Axioms A, B, C, D below. It may be helpful to point out that a symmetric variational problem in parametric form will satisfy these axioms when the extremals are considered as geodesics. It will appear that there are only two different types of spaces in which the geodesic through any two distinct points is unique, similar to the two familiar examples of the euclidean and the elliptic spaces. Namely, either the space is simply connected and all geodesics are congruent to a euclidean straight line, or the space $\Sigma$ is not simply connected and all geodesics are congruent to one euclidean circle (with the length of the shorter arc as distance). Then $\Sigma$ possesses a two-sheeted universal covering space $\Sigma_{1}$ which also satisfies the Axioms $\mathrm{A}, \mathrm{B}, \mathrm{C}, \mathrm{D}$ and which shares the following property with an ordinary sphere. All geodesics of $\Sigma_{1}$ are congruent to one euclidean circle, and the geodesics passing through a given point $X_{1}$ of $\Sigma_{1}$ all pass through the other point $X_{1}^{\prime}$ of $\Sigma_{1}$ which lies over the same point of $\Sigma$ as $X_{1}$.

This theorem will be applied to obtain improvements on the theory of spaces with convex spheres as developed in chap. 4 of the author's Metric methods in Finsler spaces and in the Foundations of geometry( $\left.{ }^{1}\right)$. Finally the two-dimensional case will be discussed.

1. Sphere like spaces. The axioms which guarantee the existence of geodesics in the space $\Sigma$ are these:

A. $\Sigma$ is metric.

The distance of the two points $A, B$ will be denoted by $A B$. We use the notation $(A B C)$ to express that the three points $A, B, C$ are different and that $A B+B C=A C$.

B. $\Sigma$ is finitely compact, that is, any bounded sequence of points has an accumulation point.

Presented to the Society, September 13, 1943; received by the editors September 27, 1942.

(1) Annals of Mathematics Studies, no. 8, Princeton, 1942. This book will be referred to as $\mathrm{B}$. It contains the references to the literature, which will therefore be omitted here. But it should be stated explicitly that in the special case of flat spaces our main result was established long ago by Hamel (see Hamel [2] in the bibliography of B). Moreover, it has been shown already in $\mathrm{B}$ that if the geodesic through any two distinct points is unique, each single geodesic is congruent to a euclidean circle or to a euclidean straight line. 
C. $\Sigma$ is convex, that is, for $A \neq C$, there is a point $B$ with $(A B C)$.

D. Every point $P$ of $\Sigma$ has a neighborhood $\nu$ with this property: For any two points $A \neq B$ in $\nu$ and a given $\epsilon>0$ there is a positive $\delta(A, B) \leqq \epsilon$ to which there corresponds a unique point $B_{\delta}$ with

$$
\left(A B B_{\delta}\right) \text { and } B B_{\delta}=\delta .
$$

We shall now compile those consequences of Axioms A, B, C, D which will be needed in the present note. For the proofs the reader is referred to B.

A point set $\tau$ is called a segment if it is congruent to an interval $[a, b]$ of the real axis, that is, there is a mapping $t \rightarrow P(t)$ of $[a, b]$ on $\tau$ such that $P\left(t_{1}\right) P\left(t_{2}\right)=\left|t_{1}-t_{2}\right|$ for any $t_{i}$ in $[a, b] . P(t)$ is called an isometric representation of $\tau$. The points $P(a)$ and $P(b)$ are the end points of $\tau$. Any two points are the end points of at least one segment. $t(P Q)$ will denote a segment from $P$ to $Q$. The following trivial property of segments will be used frequently.

(1) If $(A B C)$ and $\sigma, \tau$ are segments $\mathrm{t}(A B)$ and $\mathrm{t}(B C)$ respectively, then $\sigma+\tau$ is a segment $\mathrm{t}(A C)$.

When the spherical neighborhood $\nu(P, \rho)$ of $P$ (consisting of those points $X$ for which $P X<\rho)$ satisfies Axiom $\mathrm{D}$ and $0<\bar{\rho} \leqq \rho / 6$ then $\nu(P, \bar{\rho})$ is a socalled standard neighborhood of $P$, that is, it has the following properties:

(2) For any two distinct points $A, B$ in $\nu(P, \bar{\rho})$ and a given $0<t<1$ there is exactly one point $C$ with $(A C B)$ and $A C=t \cdot A B$. And for any given $0<s \leqq 2 \bar{\rho}$ there is exactly one point $D$ with $(A B D)$ and $B D=s$.

A point set $g$ is called a geodesic if it is a map $t \rightarrow P(t)$ of the real axis $-\infty<t<\infty$ such that for every real $t_{0}$ a positive $\epsilon\left(t_{0}\right)$ exists for which the subarc $\left|t-t_{0}\right| \leqq \epsilon\left(t_{0}\right)$ of $P(t)$ represents a segment isometrically. $P(t)$ is called an isometric representation of $g$.

(3) For a given segment $\mathfrak{t}(P Q)$ with an isometric representation $P^{\prime}(t)$, $a \leqq t \leqq b, a<b$, there exists one and only one geodesic $g$ which contains $\mathrm{t}(P Q)$, and $g$ admits an isometric representation $P(t)$ such that $P(t)=P^{\prime}(t)$ for $a \leqq t \leqq b$.

The simplest geodesics are the straight lines. They are by definition congruent either to a euclidean straight line or to a euclidean circle. More explicitly, if $P(t)$ represents a straight line isometrically then for any real $t_{1}, t_{2}$ either

(4a) $P\left(t_{1}\right) P\left(t_{2}\right)=\left|t_{1}-t_{2}\right|$ (open straight line) or an $\alpha>0$ exists such that

(4b) $P\left(t_{1}\right) P\left(t_{2}\right)=\min \left|t_{1}-t_{2}+\nu \alpha\right|, \nu=0, \pm 1, \pm 2, \cdots($ closed straight line $)$. In the latter case $g$ is congruent to a euclidean circle of radius $\alpha / 2 \pi$. Hence $\alpha$ is called the length $\lambda(g)$ of $g$. For any point $A$ of $g$ there is exactly one point $A^{\prime}$ on $g$ (the conjugate point to $A$ on $g$ ) for which $g$ contains two segments $\mathrm{t}\left(A A^{\prime}\right)$.

The point $A^{\prime}$ may also be characterized as the point on $g$ for which no point $Y$ with $\left(A A^{\prime} Y\right)$ exists. For, if $A^{\prime} \neq \bar{A} \subset g$ then $(A \bar{A} Y)$ with $Y=A^{\prime}$. If a point $Y$ with $\left(A A^{\prime} Y\right)$ existed, it would obviously not lie on $g$. On the other 
hand, it follows from (1) that a segment $\tau$ from $A$ to $A^{\prime}$ on $g$ forms together with any segment $\mathrm{t}\left(A^{\prime} Y\right)$ a segment $\tau^{\prime}$ from $A$ to $Y$. Let $g^{\prime}$ be the geodesic that contains $\tau^{\prime}$. Then $g^{\prime} \neq g$ because $Y \nsubseteq g$. But (3) and the fact that $\tau$ is contained both in $g$ and $g^{\prime}$ would yield $g=g^{\prime}$.

A straight line contains with any two points $P, Q$ also a segment $\mathrm{t}(P Q)$. The converse holds too, so that we may state

(5) $A$ geodesic is a straight line if, and only if, with any two points $P, Q$ it also contains a segment $\mathrm{t}(P Q)$.

In this form the theorem is not in B. However the proof of Theorem 3, chap. $1, \S 2$, in $\mathrm{B}$ can easily be modified to yield this result.

As a consequence of (5) we have

(6) If there is only one geodesic through any two distinct points, then all geodesics are straight lines.

Namely, if $g$ is any geodesic and $A, B$ two different points on $g$, then a geodesic $g^{\prime}$ exists containing a given segment $\mathrm{t}(A B)$ (compare (3)). Since, by hypothesis, $g=g^{\prime}$, it follows that $g$ contains a segment $\mathrm{t}(A B)$, and hence must be a straight line by virtue of (5).

A space which satisfies the hypothesis of (6) is called an S.L. space, in particular an open S.L. space if all straight lines are open, a closed S.L. space if they are all closed.

As stated in the introduction, the main purpose of this note is to show that all S.L. spaces are either open or closed, and that in the latter case all straight lines have the same length.

In any space with Axioms $\mathrm{A}$ to $\mathrm{D}$ the straight lines have this property:

(7) If two straight lines $g$ and $h$ have two different common points $A$ and $A^{\prime}$ then either $g=h$ or else $g$ and $h$ are closed, have the same length, and $A$ and $A^{\prime}$ are conjugate on both $g$ and $h$.

For if $g$ is open or closed and $A^{\prime}$ not conjugate to $A$, then $g$ contains a point $Y$ with $\left(A A^{\prime} Y\right)$ and a segment $\sigma$ from $A^{\prime}$ to $Y$. The geodesic $h$ contains a segment $\tau$ from $A$ to $A^{\prime}$. By virtue of (1) $\tau+\sigma$ is a segment $\mathrm{t}(A Y)$. Using (3) twice we find that the geodesic which contains $\tau+\sigma$ must coincide with $h$ because it contains $\tau$ and with $g$ because it contains $\sigma$. Hence, if $g \neq h$, the points $A$ and $A^{\prime}$ are conjugate on both lines, so that $\lambda(g)=\lambda(h)=2 A A^{\prime}$.

Let the space $\Sigma$ satisfy Axioms A, B, C, D. If all geodesics of $\Sigma$ are closed straight lines and if all geodesics which pass through a given point $A$ pass through a fixed point $A^{\prime}$, the conjugate point to $A$ in $\Sigma$, we call $\Sigma$ sphere like. (The word spherical will be reserved for spaces congruent to a euclidean sphere.)

(8) Let $\Sigma$ be a sphere like space. Then all geodesics have the same length. The conjugate point $A$ to $A^{\prime}$ in $\Sigma$ is the conjugate point to $A$ on every geodesic through $A$. The mapping $A \rightarrow A^{\prime}$ of $\Sigma$ on itself is an isometry. The geodesic through $A$ and $B$ is unique, when $A$ and $B$ are not conjugate. 
The first two assertions follow from (7).

There is a geodesic $g$ through $A$ and $B$. Any geodesic $g^{\prime} \neq g$ through $A$ intersects $g$, besides at $A$, only at $A^{\prime}$, hence $g$ is the only geodesic through $A$ and $B$. If we denote generally the conjugate point to $X$ by $X^{\prime}$, the isometry of the mapping $A \rightarrow A^{\prime}$ may be written as

(9) $X Y=X^{\prime} Y^{\prime}$

For $Y=X$ or $Y=X^{\prime}$ the relation (9) is obvious. When $Y \neq X, X^{\prime}$ the points $X$ and $Y$ are not conjugate. Hence the geodesic through $X$ and $Y$ is unique and contains both $X^{\prime}$ and $Y^{\prime}$. Since $X X^{\prime}=Y Y^{\prime}$ and $g$ is a closed straight line of length $2 X X^{\prime}$ we see that $X Y=X^{\prime} Y^{\prime}$.

We show next that every sphere like space is covering space of a closed S.L. space.

THEOREM 1. The non-ordered pairs $\bar{X}=\left(X, X^{\prime}\right)$ of a sphere like space $\Sigma$ form with the metric

(10) $\overline{X Y}=\min \left(X Y, X Y^{\prime}\right)$

a closed S.L. space $\bar{\Sigma}$ in which all straight lines have length $X X^{\prime}$.

Proof. Obviously $\bar{X} \bar{X}=0$. If $\bar{X} \neq \bar{Y}$, then $X \neq Y$ and $X \neq Y^{\prime}$, hence $\bar{X} \bar{Y}>0$. Moreover (9) yields

$$
\bar{X} \bar{Y}=\min \left(X Y, X Y^{\prime}\right)=\min \left(Y X, Y X^{\prime}\right)=\bar{Y} \bar{X} .
$$

The triangle inequality

(11) $\bar{X} \bar{Y}+\bar{Y} \bar{Z} \geqq \bar{X} \bar{Z}$

follows from (9), (10) and these 4 inequalities:

$$
\begin{aligned}
& X Y+Y Z \geqq X Z, \quad X Y^{\prime}+Y Z=X^{\prime} Y+Y Z \geqq X^{\prime} Z=X Z^{\prime}, \\
& X Y+Y Z^{\prime} \geqq X Z^{\prime}, X Y^{\prime}+Y Z^{\prime}=X^{\prime} Y+Y Z^{\prime} \geqq X^{\prime} Z^{\prime}=X Z,
\end{aligned}
$$

which also show that the equality sign holds in (11) only if $X, Y, Z$ are on one geodesic in $\Sigma$.

We see that $\bar{\Sigma}$ satisfies Axiom A. Clearly, Axiom B holds too. The metrization (10) applied to the points $X$ of $\Sigma$ which belong to a fixed geodesic $g$ (the point $X^{\prime}$ lies then also on $g$ ) amounts to identifying diametrically opposite points on a circle, and yields therefore a closed straight line in $\bar{\Sigma}$ with length $X X^{\prime}$. Hence any two distinct points $\bar{X}, \bar{Y}$ in $\bar{\Sigma}$ are on at least one closed straight line. This line contains a segment from $X$ to $Y$, consequently $\bar{\Sigma}$ satisfies Axiom C.

For $X Y<X X^{\prime} / 2$ we have

(12) $\bar{X} \bar{Y}=\min \left(X Y, X Y^{\prime}\right)=\min \left(X Y, Y Y^{\prime}-X Y\right)=X Y$.

Any two points $X, Y$ of a spherical neighborhood $\nu(P, \rho)$ in $\Sigma$ have distance $X Y \leqq X P+P Y<2 \rho$. Therefore it follows from (12) that the mapping $X \rightarrow \bar{X}$ maps the neighborhood $\nu\left(P, X X^{\prime} / 4\right)$ of $P$ in $\Sigma$ isometrically on the neighborhood $\nu\left(\bar{P}, X X^{\prime} / 4\right)$ in $\bar{\Sigma}$. Hence Axiom $\mathrm{D}$ holds in $\bar{\Sigma}$. 
The characteristic property of a geodesic to be locally a segment shows together with the local isometry of the mapping $X \rightarrow \bar{X}$ that each geodesic $\bar{g}$ of $\bar{\Sigma}$ consists of those points $\bar{X}=\left(X, X^{\prime}\right)$ for which $X$ belongs to a fixed geodesic in $\Sigma$. We have seen already that these points form a closed straight line of length $X X^{\prime}$. Since the geodesic through two not conjugate points $X$ and $Y$ in $\Sigma$ is unique, it follows that the geodesic through $\bar{X}$ and $\bar{Y}$ in $\bar{\Sigma}$ is unique, as soon as $\bar{X} \neq \bar{Y}$.

By means of Theorem 1 results on closed S.L. spaces lead to results on sphere like spaces. For instance Theorems 7 and 8 in B, chap. 5, $\$ 1$, yield the following:

A sphere like space is spherical (that is, congruent to a euclidean sphere) if it can be reflected in each of its straight lines.

A symmetric sphere like space is spherical.

2. The main theorem. It is easy to see that an open S.L. space is simply connected. The segment $\mathrm{t}(X Y)$ connecting two arbitrary points $X$ and $Y$ is unique and depends continuously on the end points $X$ and $Y$. Let $X(t)$, $a \leqq t \leqq b$, be any continuous curve and $P$ any point. We denote by $X_{\alpha}(t)$, $0 \leqq \alpha \leqq 1$, the unique point on $\mathrm{t}(P X(t))$ for which $P X_{\alpha}(t)=\alpha P X(t)$. The point $X_{\alpha}(t)$ depends continuously on both $t$ and $\alpha$, moreover $X_{1}(t)=X(t), X_{0}(t)=P$. Hence varying $\alpha$ from 1 to 0 we obtain a deformation of $X(t)$ into $P$.

Similarly we see that a sphere like space $\Sigma$ is simply connected. For let $X(t), a \leqq t \leqq b$, be any continuous curve which does not cover all of $\Sigma$, and $P^{\prime}$ any point not on $X(t)$; finally let $P$ be the conjugate point to $P^{\prime}$. Then the segment $\mathrm{t}(P X(t))$ is unique for every $X(t)$ and we may proceed as before.

We are going to show that an S.L. space $\bar{\Sigma}$ which is not open is not simply connected. This may be accomplished either by showing that a closed straight line cannot be contracted to a point, or by constructing a covering space $\Sigma$ different from $\bar{\Sigma}$. We shall use the second method because it will yield directly a two-sheeted sphere like space $\Sigma$. Since on the one hand 2 is the minimum number of sheets and on the other hand a sphere like space is simply connected, we see that $\Sigma$ is, topologically, the only covering space of $\bar{\Sigma}$. The original space is obtained from $\Sigma$ as in Theorem 1, which implies that all geodesics in $\bar{\Sigma}$ are closed straight lines of the same length.

Let the S.L. space $\bar{\Sigma}$ contain at least one closed geodesic $\bar{g}$, and let $\bar{A}$ be an arbitrary point on $\bar{g}$. The unique geodesic through the two different points $\bar{X}, \bar{Y}$ of $\bar{\Sigma}$ will be denoted by $\mathfrak{g}(\bar{X} \bar{Y})$. When $\bar{D} \neq \bar{A}$ and $\mathfrak{g}(\bar{A} \bar{D})$ is closed, then any geodesic $\mathfrak{g}(\bar{A} \bar{X})$ with $\bar{X} \subset \nu(\bar{D}, \epsilon)$ is closed provided $\epsilon>0$ is sufficiently small (compare $B$, chap. $3, \S 1$, Theorem 3 ).

Call $\bar{\beta}$ the locus of the conjugate points to $\bar{A}$ on the different closed geodesics through $\bar{A}$. Finally let $\nu(\bar{A}, \rho)$ be a standard neighborhood of $\bar{A}$ (compare (2)) and $\bar{\kappa}$ its boundary, that is, the locus $\bar{X} \bar{A}=\rho$.

We are going to define a mapping $\bar{X} \rightarrow X^{*}$ of $\bar{\Sigma}-\bar{\beta}$ on $\nu(\bar{A}, \rho)$. We put $A^{*}=\bar{A}$. If $\bar{A} \neq \bar{X} \subset \bar{\Sigma}-\bar{\beta}$, the segment $\mathrm{t}(\bar{A} \bar{X})$ is unique, hence there, is exactly 
one point $X^{*}$ with $\left(A X^{*} \bar{X}\right)$ and

$$
A^{*} X^{*}=\rho(\bar{A} \bar{X} /(1+\bar{A} \bar{X}))((2+\lambda \mathfrak{g}(\bar{A} \bar{X})) / \lambda \mathfrak{g}(\bar{A} \bar{X})),
$$

where $\lambda \mathfrak{g}(\bar{A} \bar{X})$ is the length of the geodesic $\mathfrak{g}(\bar{A} \bar{X})$ and

$$
(2+\lambda \mathfrak{g}(\bar{A} \bar{X})) / \lambda \mathfrak{g}(\bar{A} \bar{X})=1 \text { if } \lambda \mathfrak{g}(\bar{A} \bar{X})=\infty .
$$

Because the length of a geodesic depends continuously on the geodesic (see B, chap. $3, \S 1$ ) the mapping $\bar{X} \rightarrow X^{*}$ is one-to-one and continuous. Call $\kappa^{*}$ the subset of those points $X^{*}$ of $\bar{\kappa}$ for which $\mathfrak{g}\left(\bar{A} X^{*}\right)$ is closed. We map the point $X^{*}$ of $\kappa^{*}$ on the conjugate point $\bar{X}$ to $\bar{A}$ on $\mathfrak{g}\left(\bar{A} X^{*}\right)$. We then obtain a continuous mapping $\alpha$ of $\Sigma^{*}=\nu(\bar{A}, \rho)+\kappa^{*}$ on all of $\bar{\Sigma}$. Each point $\bar{X}$ of $\bar{\beta}$ has two images $X_{1}^{*}$ and $X_{2}^{*}$, which are diametrically opposite points of $\bar{\kappa}$.

We now assign a definite neighborhood $u\left(X^{*}\right)$ to every point of $\Sigma^{*}$ as follows: If $\bar{X}$ is not on $\bar{\beta}$ we let $u(\bar{X})$ be a standard neighborhood of $\bar{X}$ which does not meet $\bar{\beta}$, and $u\left(X^{*}\right)$ the image $u(\bar{X}) \alpha^{-1}$ of $u(\bar{X})$. If $\bar{X} \subset \bar{\beta}$ and $X_{1}^{*}$ and $X_{2}^{*}$ are its two images we choose a positive $\eta<\rho / 2$ so that $\mathfrak{g}(\bar{A} \bar{Z})$ is closed for $\bar{Z} \subset \nu\left(X_{1}^{*}, \eta\right)$ or $\bar{Z} \subset \nu\left(X_{2}^{*}, \eta\right)$. We choose a standard neighborhood $u(\bar{X})$ of $\bar{X}$ such that $g(\bar{A} \bar{Y})$ meets $\nu\left(X_{1}^{*}, \eta\right)$ and $\nu\left(X_{2}^{*}, \eta\right)$ when $\bar{Y} \subset u(\bar{X})$. (This is possible because $\mathfrak{g}\left(\bar{A} \bar{X}_{\nu}\right) \rightarrow \mathfrak{g}(\bar{A} \bar{X})$ when $\bar{X}_{\nu} \rightarrow \bar{X}$.) Let $\bar{u}_{i}, i=1,2$, be the set of those points $\bar{Y}$ in $u(\bar{X})$ for which a point $\bar{Z}_{i}$ in $\nu\left(X_{i}^{*}, \eta\right)$ with $\left(\bar{Y} \bar{Z}_{i} \bar{A}\right)$ exists. We have $\bar{u}_{1}+\bar{u}_{2}=u(\bar{X})$, and $\bar{u}_{1}$ and $\bar{u}_{2}$ have only the points $\bar{Y}$ of $\bar{\beta} \cdot u(\bar{X})$ in common. For if $\left(\bar{Y} \bar{Z}_{i} \bar{A}\right)$ and $\bar{Z}_{i} \subset \nu\left(X_{i}^{*}, \eta\right), i=1,2$, then

$$
\bar{Z}_{1} \bar{Z}_{2}>X_{1}^{*} X_{2}^{*}-2 \eta=2 \rho-2 \eta>\rho>2 \eta \text {. }
$$

If $\bar{Y}$ were not conjugate to $\bar{A}$, the segment $\mathrm{t}(\overline{A Y})$ would be unique and contain both $\bar{Z}_{1}$ and $\bar{Z}_{2}$. But then

$$
\bar{Z}_{1} \bar{Z}_{2}=\left|\bar{A}_{1}-\bar{A} \bar{Z}_{2}\right|<(\rho+\eta)-(\rho-\eta)=2 \eta .
$$

The image $u\left(X_{i}^{*}\right)$ of $\bar{u}_{i}$ we define as neighborhood of $X_{i}^{*}$.

Now let $\Sigma^{* *}$ be congruent to $\Sigma^{*}$ and designate by $X^{* *}$ the image of $X^{*}$ under a definite congruent mapping $\mu$ of $\Sigma^{*}$ on $\Sigma^{* *}$. Let $X_{1}^{*}$ and $X_{2}^{*}$ be any two diametrically opposite points of $\kappa^{*}$. We then identify $X_{1}^{*}$ with $X_{2}^{* *}$ and $X_{1}^{* *}$ with $X_{2}^{*}$. The sum of $\Sigma^{*}$ and $\Sigma^{* *}$ will be called $\Sigma$. The mappings $\alpha$ and $\mu \alpha$ define together a continuous mapping $\alpha$ of $\Sigma$ on $\bar{\Sigma}$. Under $\alpha^{-1}$ every point $\bar{X}$ of $\bar{\Sigma}$ has exactly two images $X^{*}$ and $X^{* *}$ in $\Sigma$. We say that $X^{*}$ and $X^{* *}$ lie over $\bar{X}$.

With every point of $\Sigma$ we also associate a neighborhood. Namely if $\bar{X} \Phi \bar{\beta}$ then we take the previously defined $u\left(X^{*}\right)$ as neighborhood of $X^{*}$. and $u\left(X^{* *}\right)=u\left(X^{*}\right) \mu$ as neighborhood of $X^{* *}$. If $\bar{X} \subset \bar{\beta}$ we take $u\left(X_{1}^{*}\right) \mu+u\left(X_{2}^{*}\right)$ as neighborhood of $X_{1}^{* *}=X_{2}^{*}$ and $u\left(X_{1}^{*}\right)+u\left(X_{2}^{*}\right) \mu$ as neighborhood of $X_{1}^{*}=X_{2}^{* *}$. The neighborhoods $u\left(X^{*}\right)$ and $u\left(X^{* *}\right)$ of the two points $X^{*}$ and $X^{* *}$ over $\bar{X}$ are disjoint and $\alpha$ maps both $u\left(X^{*}\right)$ and $u\left(X^{* *}\right)$ homeomorphically on the standard neighborhood $u(\bar{X})$ of $\bar{X}$. 
We metrize $\Sigma$ in the following manner: For a continuous curve $c$ with a representation $P(t), 0 \leqq t \leqq 1$, in $\Sigma$ we define the number $\lambda(c)$ as the length of the image $\bar{P}(t)=P(t) \alpha$ in $\bar{\Sigma}$ and put for any two points $X, Y$ in $\Sigma$

(13) $X Y=\inf \lambda\left(c_{X Y}\right)$,

where $c_{X Y}$ traverses all continuous curves from $X$ to $Y . X Y$ is always defined and finite. For, any point of $X^{*}$ of $\Sigma^{*}$ (or $X^{* *}$ of $\Sigma^{* *}$ ) can be connected to $A^{*}$ (or $A^{* *}$ ) by a path whose image is one of the possibly two segments from $\bar{X}$ to $\bar{A}$, and the points $A^{*}$ and $A^{* *}$ can be connected by a curve whose image is a given closed straight line through $\bar{A}$ in $\bar{\Sigma}$.

The relations $X X=0, X Y=Y X, X Y+Y Z \geqq X Z$ are obvious and so is $X Y>0$, for $\bar{X} \neq \bar{Y}$ (we put generally $Z \alpha=\bar{Z}$ ). If $\bar{X}=\bar{Y}$ the relation $X Y>0$ follows from $u\left(X^{*}\right) \cdot u\left(X^{* *}\right)=0$.

We show next that with this metrization of $\Sigma$ the mapping $\alpha$ is locally isometric. We see from the definition of $\lambda(c)$ and $X Y$ that

(14) $X Y \geqq \bar{X} \bar{Y}$.

If $u(\bar{X})=\nu(\bar{X}, \delta)$ we put $\nu(\bar{X})=\nu(\bar{X}, \delta / 2)$. Under the homeomorphic mapping $\alpha$ of $u(X)$ on $u(\bar{X})$ where $X$ stands for a definite one of the two images of $\bar{X}$, the set $\nu(X)$ may correspond to $\nu(\bar{X})$. Let $A, B \subset \nu(X)$. Since $\bar{A}, \bar{B} \subset \nu(\bar{X})$ the (unique) segment $\mathrm{t}(\overline{A B})$ lies completely in $u(\bar{X})$ so that the image of $\mathrm{t}(\overline{A B})$ in $u(X)$ has the same length as $\mathrm{t}(\overline{A B})$, that is $\overline{A B}$. Hence it follows from (13) and (14) that

(15) $A B=\overline{A B}$ for $A, B \subset \nu(X)$.

We conclude from $(15)$ that $\nu(X)$ satisfies the requirements of $D$ for the point $X$; since $X$ was an arbitrary point of $\Sigma$ we see that Axiom $\mathrm{D}$ holds.

Because of the local isometry (15) the number $\lambda(c)$ equals the length of $c$ as curve in $\Sigma$.

Axiom B now follows readily. For if $P X_{\nu}<d$ then $\bar{P} \bar{X}_{\nu}<d$ because of (14). Hence $\left\{\bar{X}_{\nu}\right\}$ contains a subsequence $\left\{\bar{X}_{\nu_{n}}\right\}$ converging to a point $\bar{X}$ in $\bar{\Sigma}$. (15) implies that with proper notations the images $\left\{X_{\nu_{n}}^{*}\right\}$ and $\left\{X_{\nu_{n}}^{* *}\right\}$ of $\left\{\bar{X}_{\nu_{n}}\right\}$ tend to the images $X^{*}$ and $X^{* *}$ of $\bar{X}$ respectively. The sequence $\left\{X_{\nu_{n}}\right\}$ contains infinitely many points of at least one of the two sequences $\left\{X_{\nu_{n}}{ }^{*}\right\}$ and $\left\{X_{\nu_{n}}^{* *}\right\}$; which proves Axiom B.

To prove Axiom $C$ we observe that because of $B$ and (13) a curve $c_{X Z}$ exists which connects two given points $X$ and $Z$ and such that

$$
X Z=\lambda\left(c_{X Z}\right)
$$

(compare Property Le in B, chap. $1, \S 1$ ). The additivity of the arclength and the triangle inequality yield in the well known fashion that $X Y+Y Z=X Z$ for every point of $c_{X Z}$.

We conclude from the local isometry (15) of $\Sigma$ and $\bar{\Sigma}$ that the image $\bar{g}=g \alpha$ of any geodesic in $\Sigma$ is a geodesic in $\bar{\Sigma}$, and that at least one geodesic $g$ in $\Sigma$ lies over every geodesic $\bar{g}$ in $\bar{\Sigma}$.

Moreover, if $g$ and $h$ both lie over $\bar{g}$ and have a common point $P$, then 
$g=h$, because $g$ and $h$ coincide in a neighborhood of $P$ (compare (3)). It follows that there is only one geodesic which lies over a given geodesic $\bar{g}$ of $\bar{\Sigma}$. For if $g_{1}$ and $g_{2}$ lie over $\bar{g}$ we select a point $G_{i}$ on $g_{i}$ such that $\bar{G}_{1} \neq \bar{G}_{2}$. There is a geodesic $k$ through $G_{1}$ and $G_{2}$. Its image $\bar{k}$ intersects $\bar{g}$ at both $\bar{G}_{1}$ and $\bar{G}_{2}$, hence $\bar{g}=\bar{k}$, and it follows from $k \cdot g_{i} \supset G_{i}$ that $k=g_{i}$ so that also $g_{1}=g_{2}$. We may now conclude

(16) If a geodesic $g$ of $\Sigma$ contains $X^{*}$, then $g$ also contains $X^{* *}$.

For $g$ lies over a geodesic $\bar{g}$ through $\bar{X}$, and there is a geodesic $g_{1}$ over $\bar{g}$ through $X^{* *}$. Hence $g=g_{1} \supset X^{* *}$. Before we apply this result to the present problem we notice a consequence which will be important in the theory of the convexity of spheres.

THEOREM 2. In a closed S.L. space each geodesic $\bar{g}$ intersects the locus $\beta(\bar{A})$ of the conjugate points to an arbitrary point $\bar{A}$ (possibly $\bar{g} \subset \beta(\bar{A})$ ).

Returning to our spaces $\Sigma$ and $\bar{\Sigma}$ we see that the geodesics through $\bar{A}$ must all be closed, for an open geodesic would have two images, one in the interior of $\Sigma^{*}$ and one in $\Sigma^{* *}$.

Since the length $\lambda(\bar{g})$ of a geodesic $g$ depends continuously on $\bar{g}$, and all geodesics through $\bar{A}$ are closed, the lengths of the geodesics through $\bar{A}$ are bounded. Hence the space $\bar{\Sigma}$ is bounded and therefore compact. We show next

(17) Each geodesic $g$ in $\Sigma$ is a closed straight line.

Since $\bar{\Sigma}$ is compact $\Sigma$ is compact, hence open straight lines cannot exist. Because of $(5)$ it is sufficient to show that $g$ contains with any two points $X, Y$ also a segment $\mathrm{t}(X Y)$. Let $\bar{X} \neq \bar{Y}$. Consider a segment $\mathrm{t}(X Y)$ and a geodesic $h$ which contains this segment $\mathrm{t}(X Y)$. Then $\bar{g}$ and $\bar{h}$ both contain $\bar{X}$ and $\bar{Y}$, hence $\bar{g}=\bar{h}$ and $g=h$, so that $g$ contains $\mathrm{t}(X Y)$. Now let $\bar{X}=\bar{Y}$, and consider a sequence of points $Y_{\nu} \neq Y$ on $g$ which tend to $Y$. For large $\nu$ we have $\bar{Y}_{\nu} \neq \bar{X}$. Hence $g$ contains a segment $\mathrm{t}\left(X Y_{\nu}\right)$. A suitable subsequence of $\left\{\mathrm{t}\left(X Y_{\nu}\right)\right\}$ tends to a segment $\mathrm{t}(X Y)$. As the image of $\bar{g}$ the geodesic $g$ is closed as a point set, hence $\mathrm{t}(X Y) \subset \mathrm{g}$, which proves (17).

(16) and (17) show that $\Sigma$ is a sphere like space. Moreover $\bar{\Sigma}$ can be obtained from $\Sigma$ as in Theorem 1. This implies that all geodesics in $\bar{\Sigma}$ have length $X^{*} X^{* *}$. We formulate our results as follows:

Theorem 3. Let the space $\bar{\Sigma}$ satisfy Axioms A, B, C, D and let there be only one geodesic through two distinct points of $\bar{\Sigma}$.

Then $\bar{\Sigma}$ is either simply connected and all geodesics are congruent to euclidean straight lines.

Or $\bar{\Sigma}$ has a sphere like space $\Sigma$ as two-sheeted universal covering space, from which $\bar{\Sigma}$ is obtained by identifying conjugate points. The geodesics of $\bar{\Sigma}$ are all congruent to euclidean circles and have the same length.

3. Convexity of spheres. A sphere $K(A, \rho)$ is the locus of those points 
$X$ for which $X A=\rho$. The set $\nu(A, \rho)$ is by definition the interior of $K(A, \rho)$. When $\alpha$ is the common length of the straight lines of a closed S.L. space $K(A, \alpha / 2)$ is the locus $\beta(A)$ of the conjugate points to $A$, and $K(A, \rho)$ is empty for $\rho>\alpha / 2$. In the following the radius $\rho$ of a sphere is always assumed to be not greater than $\alpha / 2$.

Theorems 2 and 3 enable us to simplify and to complete the theory of spaces with convex spheres as developed in B, chap. 4 . We shall indicate the new method here.

In an open S.L. space we would naturally say that $K(A, \rho)$ is convex if a segment $\mathrm{t}(X Y)$ whose end points $X, Y$ are in or on $K(A, \rho)$ lies except for $X, Y$ in the interior of $K(A, \rho)$. But this definition cannot be applied to closed S.L. spaces, since then not even all spheres of an elliptic space would be convex. In euclidean geometry an equivalent definition of convexity is available in terms of supporting planes. One is therefore led to ask whether convexity can be expressed satisfactorily in terms of the existence of supporting geodesics.

We call the straight line $t$ of a sphere like space or an S.L. space a tangent of $K(A, \rho)$ at $Z$ if points $X_{\nu} \neq Y_{\nu}$ on $K(A, \rho)$ exist which tend to $Z$ and such that the line $\mathfrak{g}\left(X_{\nu} Y_{\nu}\right)$ tends to $t$. We say that $K(A, \rho)$ is convex if no tangent of $K(A, \rho)$ contains points of $\nu(A, \rho)$. The spheres of an elliptic space are convex in this sense.

On the other hand one might consider dropping the distinction between the interior and exterior of a sphere and simply require that the spheres are surfaces of order 2 . More exactly we say that a set $\mu$ of a sphere like space or of an S.L. space has order not greater than $n$ if any straight line intersects $\mu$ in at most $n$ different points, or else is contained in $\mu$, and that $\mu$ has order $n$ if it has order not greater than $n$ but not order less than or equal to $n-1$. We can then show the following:

(18) Let the sphere $K(A, \rho)$ of an arbitrary S.L. space $\Sigma$ not be the locus $\beta(A)$ of the conjugate points to $A$ and have order 2 . Then $K(A, \rho)$ is convex.

Assume for an indirect proof that a point $Z$ on $K(A, \rho)$ and a tangent $t$ of $K(A, \rho)$ at $Z$, which contains a point $P$ of $\nu(A, \rho)$, exist. By definition $t$ is limit of a sequence of straight lines $\mathfrak{g}\left(X_{\nu} Y_{\nu}\right)$ where $X_{\nu} \neq Y_{\nu}, X_{\nu} A=Y_{\nu} A=\rho$ and $X_{\nu} \rightarrow Z, Y_{\nu} \rightarrow Z$. Choose $P_{\nu}$ on $\mathfrak{g}\left(X_{\nu} Y_{\nu}\right)$ such that $P_{\nu} \rightarrow P$.

First let $\Sigma$ be an open S.L. space. Then either of the subrays of $\mathfrak{g}\left(X_{\nu} Y_{\nu}\right)$ with origin $P_{\nu}$ contains a point of $K(A, \rho)$ (because $P T$ varies continuously as $T$ traverses $g\left(X_{\nu} Y_{\nu}\right)$ ). The ray which contains $X_{\nu}$ will also contain $Y_{\nu}$, at least for large $\nu$, because $X_{\nu} Y_{\nu} \rightarrow 0$. But then $\mathfrak{g}\left(X_{\nu} Y_{\nu}\right)$ would intersect $K(A, \rho)$ in at least three points.

Next let $\Sigma$ be closed and $K(A, \rho) \neq \beta(A)$. Because of Theorem 2 the line $\mathfrak{g}\left(X_{\nu} Y_{\nu}\right)$ contains a point $Q_{\nu}$ of $\beta(A)$. Then $Q_{\nu} A>\rho$. For the same reason as before either subarc of $\mathfrak{g}\left(X_{\nu} Y_{\nu}\right)$ from $P_{\nu}$ to $Q_{\nu}$ contains a point of $K(A, \rho)$, 
and for large $\nu$ the arc which contains $X_{\nu}$ also contains $Y_{\nu}$, so that $\mathrm{g}\left(X_{\nu} Y_{\nu}\right)$ would intersect $K(A, \rho)$ in at least three different points. This result will be completed by:

(19) If the spheres $K(A, \rho)$ of a closed S.L. space have order not greater than 2 then $\beta(A)$ has order 1 .

We have to show that $\beta(A)$ contains the line $g$ if it contains the two distinct points $X$ and $Y$ of $g$. Call $\delta_{1}$ and $\delta_{2}$ the two subarcs of $g$ from $X$.to $Y$. If the theorem were not true, at least one of the arcs, $\delta_{1}$ say, would contain a point $P$ with $P A=\rho-\epsilon_{1}, \epsilon_{1}>0$. Then $\delta_{2}$ cannot contain a point $Q$ with $Q A=\rho-\epsilon_{2}, \epsilon_{2}>0$, brecause the sphere $K\left(A, \rho-\min \left(\epsilon_{1}, \epsilon_{2}\right) / 2\right)$ would contain at least four points of $g$ without containing $g$. Hence $K(A, \alpha / 2)=\beta(A)$ would have to contain $\delta_{2}$ without containing $g$, and would not have order less than or equal to 2 .

The results (18) and (19) lead to the following theorem:

THEOREM 4. In any S.L. space the following properties are equivalent:

I. The spheres are convex.

II. The spheres have order not greater than 2.

III. A given point $P$ has exactly one foot on a given straight line $g$; or else $P X$ is constant for $X \subset g$.

IV. If the point $P$ does not lie on the line $g$, then either $P X=$ const. for $X \subset g$ and all lines $\mathfrak{g}(P X)$ are perpendicular $\left(^{2}\right)$ to $g$, or else there is exactly one perpendicular through $P$ to $g$.

A proof of Theorem 4 may be obtained by combining our present results with the results in $B$, chap. $4, \S \S 1$ and 3 . Although many simplifications are now possible we refrain from giving a complete proof here in order to avoid duplication.

B, chap. 4, 1 , Theorem 3, states that I implies III, and B, chap. 4, §3, Theorem 1 contains the equivalence of II and III. Our present relations (18) and (19) show that II implies I. Finally B, chap. 4, §3, Theorems 4, 5 and 6 yield together with the present Theorems 2 and 3 that IV follows from II. Therefore we have to show here only that IV implies III. Let $P$ not be conjugate to all points of $g$ and not on $g$ (otherwise III is trivial) and consider the perpendicular $g(P F), F \subset g$, through $P$ to $g$. Since not all points of $g$ are conjugate to $P, F$ (as a foot of $P$ ) is not conjugate to $P$, so that $g(P F)$ contains a point $Q$ with $(Q P F)$. According to the definition of a perpendicular $F$ is a foot of $Q$ on $g$, therefore we have for every $X \neq F$ on $g$

$$
P X>Q X-Q P \geqq Q F-Q P=P F,
$$

which means that $F$ is the only foot of $P$ on $g$.

(2) The straight line $h \neq g$ is a perpendicular to $g$ if $h$ intersects $g$ and all points of $h$ have this intersection as foot on $g$. 
Theorems 1 and 4 yield, together with $\mathrm{B}$, chap. 4 , $\$ 2$, Theorem 1 , the following characterization of the elliptic spaces.

Theorem 5a. A closed S.L. space of dimension not less than 3, which has one of the properties I, II, III, or IV, is congruent to a finite dimensional elliptic space.

A sphere $K(A, \rho)$ of a sphere like space of dimension not less than 2 whose geodesics have length $2 \alpha$ is not convex as soon as $\rho>\alpha / 2$. But the point sets which carry $K(A, \rho)$ and $K\left(A^{\prime}, \alpha-\rho\right)$ coincide and one of these spheres is convex. Therefore we conclude from Theorems $5 \mathrm{a}$ and 1 :

ThEOREM 5b. Let $\Sigma$ be a sphere like space of dimension not less than 3, whose geodesics have length $2 \alpha$. If the spheres of radius $\rho \leqq \alpha / 2$ are convex, or if one of the properties II, III, IV holds, $\Sigma$ is congruent to a euclidean sphere.

In $\mathrm{B}$, chap. $4, \S 2$ an example of Hamel was quoted to show that a closed S.L. space is not even then necessarily elliptic when its geodesics are the straight lines of a projective space. By means of Theorem 1 we see from this example that there are metrizations of a euclidean sphere (of any dimension not less than 2) in which the geodesics are the euclidean great circles but the metric is not spherical. The condition of Theorem $5 \mathrm{~b}$ may therefore not be omitted.

4. Two-dimensional spaces. The characterization (5) of the straight lines suggests the problem to determine all spaces which satisfy Axioms A, B, C, D and in which the geodesics are straight lines. As solution one would conjecture the S.L. spaces and the sphere like spaces. The proof seems difficult for higher dimensions but it is easy for two.

THEOREM 6. Let each geodesic of a two-dimensional space $\Sigma$ (with Axioms $\mathrm{A}, \mathrm{B}, \mathrm{C}, \mathrm{D})$ contain a segment $\mathrm{t}(X Y)$ when it contains $X$ and $Y$. Then $\Sigma$ is either homeomorphic to the euclidean plane and an open S.L. space, or homeomorphic to the projective plane and a closed S.L. space, or homeomorphic to a 2-sphere and sphere like.

Proof. If no two geodesics have more than one common point, the assertion is contained in $\mathrm{B}$, chap. $3, \S 2$, Theorem 1 . Let therefore two different geodesics $g$ and $h$ which have the two common points $A$ and $A^{\prime}$ exist. It follows from (7) that they have no further common points and that $g$ and $h$ are closed with $A$ and $A^{\prime}$ as conjugate points on both lines.

Call $\sigma_{1}, \sigma_{2}$ and $\tau_{1}, \tau_{2}$ the two segments $\mathfrak{t}\left(A A^{\prime}\right)$ on $g$ and $h$ respectively. Because of $\mathrm{B}$, chap. $1, \S 4$, Theorem 4 , a standard neighborhood $\nu(A, \rho)$ of $A$ is homeomorphic to a circular disk. Let $S_{i}, T_{j}$ be the point on $\sigma_{i}$ and $\tau_{j}$ respectively with $S_{i} A=T_{j} A=\rho$, and call $g_{X}$ a geodesic which carries a segment $\mathrm{t}\left(A^{\prime} X\right)$, where $X$ is an interior point of the unique segment $\mathrm{t}\left(S_{i} T_{j}\right)$. By virtue of $\mathrm{B}$, chap. $1, \S 4$, Theorem $3, g_{X}$ intersects $\mathrm{t}\left(S_{i} A\right)+\mathfrak{t}\left(A T_{j}\right)$. The point $A$ is 
the only possible intersection because each intersection of $g_{X}$ with $g$ or $h$ other than $A^{\prime}$ is conjugate to $A^{\prime}$ on $g_{X}$ and $g$ or $h$.

Therefore the (unique) segment $\mathrm{t}(A X)$ lies on $g_{X}$. The set $\sum_{x} \mathrm{t}(A X)$ with $X \subset \sum_{i, j} \mathrm{t}\left(S_{i} T_{j}\right)$ covers a whole neighborhood of $A$, hence all geodesics through $A$ are closed straight lines and pass through $A^{\prime}$. The geodesic $\mathrm{g}(A X)$ is unique because $X$ is not conjugate to $A$, and varies continuously with $X$. Hence the space is homeomorphic to the 2 -sphere.

Consider now any point $B \neq A, A^{\prime}$. There is a geodesic $k$ through $A$ and $B$. Let $l$ be any other geodesic through $B$. Since $l$ crosses $k$ at $B$ and is a closed Jordan curve it follows from the topological structure of a sphere that $l$ intersects $k$ at some other point $B^{\prime}$. We see as before that every geodesic through $B^{\prime}$ passes also through $B$. Since $B$ was arbitrary the space is sphere like.

In an elliptic space we have complete duality between point and hyperplane. The dual of a given theorem is obtained by interchanging a point $A$ with the hyperplane $\beta(A)$ formed by the conjugate points to $A$. In a general closed S.L. space of dimension $n \geqq 3$, hyperplanes are not defined and questions of duality become void.

But we may ask whether there are duality relations which hold in every closed S.L. plane. $\beta(A)$ will in general not be a straight line. But in the elliptic case $\beta(A)$ may be characterized as the straight line which has maximal distance from $A$. We shall show the following theorem:

THEOREM 7. For every point $\bar{A}$ of a closed S.L. plane $\bar{\Sigma}$ there is exactly one straight line $g(\bar{A})$ which has maximal distance from $\bar{A}$. The correspondence $\bar{A} \rightarrow g(\bar{A})$ maps $\bar{\Sigma}$ topologically on the set $\pi$ of all geodesics in $\bar{\Sigma}$.

By Theorem 3 this theorem is equivalent to the following statement on a two-dimensional sphere like space $\Sigma$.

For a given point $A$ in $\Sigma$ there is exactly one straight line $g(A)$ which has maximal distance from $A$. When $A^{\prime}$ is conjugate to $A$, then $g(A)=g\left(A^{\prime}\right)$. The mapping $\left(A, A^{\prime}\right) \rightarrow g(A)$ of the pairs of conjugate points on the geodesics in $\Sigma$ is topological.

We shall prove this fact. Because of Theorem 6 the space $\Sigma$ is homeomorphic to the 2 -sphere. Designate by $4 \alpha$ the common length of the geodesics in $\Sigma$ and by $d(A, g)$ the distance of the point $A$ from the geodesic $g$. If $A$ is not conjugate to $X$ then the line $\mathfrak{g}(A X)$ is unique for $X \subset g$ and intersects $g$ also in $X^{\prime}$. We see from

(20) $2 \alpha=X A+A X^{\prime}=X^{\prime} A^{\prime}+A^{\prime} X$ that

(21) $d(A, g)=d\left(A^{\prime}, g\right)$

and $\min \left(A X, A X^{\prime}\right) \leqq \alpha$, hence

(22) $d(A, g) \leqq \alpha$. The equality sign holds if and only if $A X \equiv \alpha$ for $X \subset g$.

The number $\bar{d}(A, g)$ depends continuously on $A$ and $g$. For a given point $A$ there is a line $g_{0}$ for which $d(A, g)$ reaches its maximum $m \leqq \alpha$. 
(23) $d\left(A, g_{0}\right)=m=\alpha$ if and only if $A$ has two feet on $g_{0}$ which are conjugate to each other.

For if $F$ and $F^{\prime}$ are feet of $A$ on $g_{0}$, we see from (20) that $d\left(A, g_{0}\right)=A F$ $=A F^{\prime}=\alpha$. Conversely if $d\left(A, g_{0}\right)=\alpha$, then $A X=A X^{\prime}=\alpha$ for any $X$ on $g_{0}$ because of (22).

The main point of the proof is

(24) If $g_{0}$ has maximal distance from $A$, then any open segment on $g_{0}$ of length $2 \alpha$ contains a foot of $A$.

Assume for an indirect proof that (24) is not true. Then it follows from (23) that $g_{0}$ contains no two conjugate feet. Since the feet of $A$ form a closed subset of $g_{0}$, there is a closed segment $s$ of length $2 \alpha$ on $g_{0}$ which contains no foot of $A$, so that $A X>m+\epsilon$ for $X \subset s$ and a suitable $\epsilon>0$.

The endpoints $Y, Y^{\prime}$ of $s$ are conjugate. Call $s^{\prime}$ the other segment $\mathrm{t}\left(Y Y^{\prime}\right)$ on $g_{0}$. A foot $F$ of $A$ is an interior point of $s^{\prime}$. Since $A F=m<\alpha$, there are points $Z$ with $(A F Z)$. The line $g_{Z}=\mathfrak{g}(Y Z)$ is unique and passes through $Y^{\prime}$. Call $s_{Z}^{\prime}$ the subsegment $\mathrm{t}\left(Y Y^{\prime}\right)$ of $g_{Z}$ which contains $Z$ and $s_{Z}$ the other subsegment $\mathrm{t}\left(Y Y^{\prime}\right)$ of $g_{Z}$. For $Z \rightarrow F$ we have $g_{Z} \rightarrow g_{0}, s_{Z} \rightarrow s, s_{Z}^{\prime} \rightarrow s^{\prime}$ and $d\left(A, g_{Z}\right) \rightarrow d\left(A, g_{0}\right)$. Consequently, when $F Z$ is sufficiently small each foot $F_{Z}$ of $A$ on $g_{Z}$ belongs to $s_{Z}^{\prime}$ so that $d\left(A, s_{Z}^{\prime}\right)=d\left(A, g_{Z}\right)$. But $s^{\prime}$ separates $A$ from $s_{Z}^{\prime}$ in the domain containing $A$ and bounded by $s$ and $s_{Z}^{\prime}$. Hence $d\left(A, g_{Z}\right)=d\left(A, s_{Z}^{\prime}\right)>d\left(A, s^{\prime}\right)$ $=d\left(A, g_{0}\right)$, which contradicts the definition of $g_{0}$.

(25) Any line $h \neq g_{0}$ contains an open segment of length $2 \alpha$ which is free from feet of $A$.

This statement is trivial for $A \subset h$. Let therefore $A$ not be on $h$ and designate by $D$ and $D^{\prime}$ the intersections of $g_{0}$ and $h$, by $\mu$ the side of $g_{0}$ which contains $A$, and by $t$ the segment $\mathrm{t}\left(D D^{\prime}\right)$ on $h$ which is not contained in $\mu$. For any interior point $G$ of $t$ the segment $\mathrm{t}(A G)$ intersects $g$ at some point $H \neq D, D^{\prime}$. Hence $G$ is no foot of $A$ on $h$, because otherwise

$$
d(A, h)=A G>A H \geqq d\left(A, g_{0}\right),
$$

which contradicts the definition of $g_{0}$. We see that the interior of $s$ satisfies (25).

(24) and (25) show that $g_{0}$ is the only line which has maximal distance from $A$. We designate this line henceforth by $g(A)$. Moreover $g(A)=g\left(A^{\prime}\right)$.

(26) If $B \neq A, A^{\prime}$ then $g(A)$ contains an open segment of length $2 \alpha$ which is free from feet of $B$.

The statement is obvious for $B \subset g(A)$. If $B$ does not lie on $g(A)$, let it lie on the same side of $g(A)$ as $A$. The line $\mathfrak{g}(A B)$ contains a point $H$ on $g(A)$ with $(A B H)$. If $H$ is a foot of $A$ on $g(A)$, then any $X \neq H$ on $g$ satisfies the inequality

$$
B X>A X-A B \geqq A G-A B=B G,
$$

so that $G$ is the only foot of $B$ on $g(A)$ and (26) holds. If $H$ is not a foot of $A$, 
we traverse $g(A)$ from $H$ on, in either direction, until we meet the first foot of $A$. We may thus obtain the feet $F_{1}, F_{2}$. Because of (24) we have $F_{1} F_{2}<2 \alpha$. To prove (26) it suffices to see that $\mathrm{t}\left(F_{1} F_{2}\right)$ contains all feet of $B$. If $G$ is any point on $g(A)-\mathrm{t}\left(F_{1} F_{2}\right)$, the segment $\mathrm{t}(A G)$ intersects $\mathrm{t}\left(A F_{1}\right)+\mathrm{t}\left(A F_{2}\right)$ at some point $R$. Let $R \subset \mathrm{t}\left(A F_{1}\right)$. Since $F_{1} F_{2}<2 \alpha$ the point $F_{1}$ is different from the conjugate point $H^{\prime}$ to $H$, hence we obtain, using that $F_{1}$ is a foot of $A$,

$B F_{1}<B R+R F_{1}=B R+A F_{1}-A R \leqq B R+A G-A R \leqq B R+G R=B G$.

This shows that $G$ is not a foot of $B$.

We conclude from (24) and (26) that $g(A) \neq g(B)$ for $A \neq B$; therefore the mapping $A \rightarrow g(A)$ is one-to-one. The continuity follows immediately from the continuity of $d(A, g)$ in $A$ and $g$.

It is easily seen that all geodesics of $\Sigma$ form a set $\pi$ homeomorphic to the projective plane. Let $\mu$ map $\pi$ topologically on $\Sigma$. The mapping $A \rightarrow g(A)$ $\rightarrow g(A) \mu$ maps $\Sigma$ on the subset $\Sigma^{\prime}$ of those points which correspond to geodesics which have maximal distance from some point of $\Sigma$. Since the mapping of $\Sigma$ on $\Sigma^{\prime}$ is topological, we have $\Sigma=\Sigma^{\prime}$. Hence $g(A)$ traverses all of $\pi$ as $A$ traverses $\Sigma$.

This theorem throws some light on the difficulties of the problem to determine the closed S.L. planes with convex circles, because it shows how weak an assumption the convexity of the circles is in two dimensions.

ILLINOIS INSTITUTE OF TECHNOLOGY, Chicago, Ill. 